\title{
AUTOMATED INSPECTION OF POWER LINE CORRIDORS TO MEASURE VEGETATION UNDERCUT USING UAV-BASED IMAGES
}

\author{
M. Maurer ${ }^{\mathrm{a}}$, M. Hofer ${ }^{\mathrm{b}}$, F. Fraundorfer ${ }^{\mathrm{a}}$, and H. Bischof ${ }^{\mathrm{a}}$ \\ ${ }^{a}$ Institute for Computer Graphics and Vision, Graz University of Technology, Austria - (maurer, fraundorfer, bischof)@icg.tugraz.at \\ ${ }^{b}$ Naked Labs GmbH, Graz, Austria - manuel@nakedlabs.com
}

KEY WORDS: 3D Reconstruction, UAV Images, Semantic Segmentation, Automated Measuring, Clearance Distance, Inspection

\begin{abstract}
:
Power line corridor inspection is a time consuming task that is performed mostly manually. As the development of UAVs made huge progress in recent years, and photogrammetric computer vision systems became well established, it is time to further automate inspection tasks. In this paper we present an automated processing pipeline to inspect vegetation undercuts of power line corridors. For this, the area of inspection is reconstructed, geo-referenced, semantically segmented and inter class distance measurements are calculated. The presented pipeline performs an automated selection of the proper 3D reconstruction method for on the one hand wiry (power line), and on the other hand solid objects (surrounding). The automated selection is realized by performing pixel-wise semantic segmentation of the input images using a Fully Convolutional Neural Network. Due to the geo-referenced semantic 3D reconstructions a documentation of areas where maintenance work has to be performed is inherently included in the distance measurements and can be extracted easily. We evaluate the influence of the semantic segmentation according to the $3 \mathrm{D}$ reconstruction and show that the automated semantic separation in wiry and dense objects of the $3 \mathrm{D}$ reconstruction routine improves the quality of the vegetation undercut inspection. We show the generalization of the semantic segmentation to datasets acquired using different acquisition routines and to varied seasons in time.
\end{abstract}

\section{INTRODUCTION}

Power line corridors have to be inspected on a regular basis according to vegetation growing too close to the power line. In industry this inspection task is known as vegetation undercut detection or clearance distance inspection. Up to now these inspections are done manually. On the one hand a person is walking by the power line corridor and estimates the distances between vegetation and power line. This leads to coarse results and most of the time hardly traceable documentation. On the other hand these inspections can be done using aerial images acquired using manned systems. Acquisition hardware of aerial images typically has very high resolution. The restrictions on minimum flight height and thus having large distance to the scene, aerial images result in ground sampling distances (GSD), like for the UltraCamEagle Mark $2^{1}$, of $27 \mathrm{~mm}$ at a flight height of $1000 \mathrm{~m}$. But the usage of aerial images is quite expensive as there is an enormous demand of resources. Compared to aerial images, images taken by an Unmanned Aerial Vehicles (UAV) also provide high GSD because of the close distance UAVs can be operated to the scene. Next to nadir views as delivered by aerial images it is easily possible to add slightly slanted views using UAVs which improves the reconstruct-ability of power lines and the side views of the surroundings (vegetation). Due to the development of UAVs in recent years we are now able to acquire high quality UAV images at low cost. The diversity in design of UAVs provides the ability to hover and perform very precise maneuvers at close distance to an object using multi-rotor UAVs, or survey large areas using fixed-wing UAVs.

Nowadays UAV images are used for different purposes: 3D reconstruction, inspection and measuring. There are commercial products available for $3 \mathrm{D}$ reconstruction like Pix $4 \mathrm{D}^{2}$ or Agisoft

\footnotetext{
${ }^{1} \mathrm{http} / / / \mathrm{www} . v e x c e l$-imaging.com/products/ultracam-eagle

${ }^{2}$ https://pix4d.com/
}

PhotoScan ${ }^{3}$. A similar system is the one by (Tscharf et al., 2015). They present a fully automated approach to generate precise, metric and geo-accurate 3D reconstructions using UAV images and terrestrial ones. High resolution UAV imagery is also used in (Jóźków et al., 2015) to model the geometry of transmission lines in 3D. Their experiment showed that having properly acquired images and using a dense matching algorithm provides similar results as using LiDAR acquired point clouds. It is convenient to use UAVs as investigation tool to acquire images for inspection tasks of otherwise hard to reach positions. One example is the inspection of roofs according to the thermal insulation. This was presented by (Zhang et al., 2015) where they propose automatic thermal anomaly detection in $2 \mathrm{D}$, based on thermal images acquired using UAVs. A further application UAVs are used for is the inspection of wetlands according to the vegetation growing there. In (Marcaccio et al., 2015) they demonstrate the usability of UAV imagery for classifying the wetland vegetation at different seasons to be able to map the seasonal changes in habitat.

The previously mentioned publications do either 3D reconstruction or inspection or measuring. But we present an approach that does combine these tasks. We want to perform analysis. In addition to pure $3 \mathrm{D}$ reconstruction we relate objects present in the $3 \mathrm{D}$ reconstruction to each other to measure interpretable distances. Therefore, we first perform semantic segmentation and reconstruct the scene. The quality of reconstruction is enhanced as we interpret the object to be reconstructed according to the semantics and automatically select a proper reconstruction routine. By transferring the semantics to the scene we gain the ability to not only perform 3D measurements, but are able to interpret them. Our approach is demonstrated on the task of inspecting power line corridors according to vegetation undercuts.

\footnotetext{
${ }^{3}$ http://www.agisoft.com/
} 


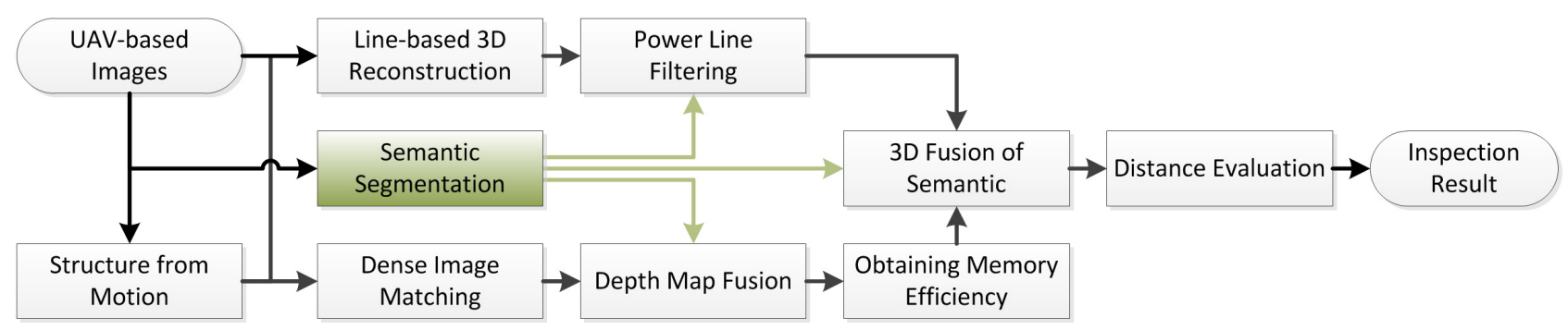

Figure 1. Overview of the automated vegetation undercut inspection pipeline: Based on UAV-based images we automatically reconstruct the area, add semantics to the 3D model and finally measure distances between power lines and trees. The core part of the

framework, the semantic segmentation, enables to interpret the object to be reconstructed according to the semantics and to automatically select the proper reconstruction routine. This means we will reconstruct the surrounding with semantically filtered power lines using a dense approach and the power lines using the line-based approach.

\section{PIPELINE}

In this section we describe our highly automated pipeline to get semantically labeled 3D reconstructions containing a dense representation of the environment and preserving wiry objects like power lines. This semantically labeled 3D reconstruction is further simplified for the reason of efficiently performing inspection tasks within semantic classes. The pipeline is depicted in Figure 1 and consists of the following main parts:

- Geo-referenced Structure-from-Motion and Line-based Reconstruction

- Semantic Segmentation

- Dense Image Matching

- Depth Map Fusion with Semantic Filtering

- Obtaining Memory Efficiency

- 3D Fusion of Semantics

- Distance Evaluation

\subsection{Geo-referenced Structure-from-Motion and Line-based Reconstruction}

The task of a Structure-from-Motion (SfM) pipeline is to reconstruct $3 \mathrm{D}$ points and simultaneously calculate the camera poses according to the reconstructed sparse point cloud. The presented approach is highly automated and is capable to deal with unordered, non-sequential image sets. In our pipeline we follow (Irschara et al., 2012) to get the sparse point cloud with oriented cameras. We assume pre-calibrated input images and we achieve this by exploiting the method of (Daftry et al., 2013). The first step of the SfM pipeline is the extraction of features. As features, Scale-invariant Feature Transform (SIFT) presented by Lowe (Lowe, 2004) is used, as they are robust against illumination, rotation and view point changes. Next the extracted features are matched to create inter image correspondences. This process is speeded up by using a vocabulary tree matching (Nister and Stewenius, 2006). Using the corresponding features between image pairs the relative motion between these two cameras is estimated and geometrically verified using a robust version to the five-point algorithm (Nistér, 2004). Based on an initial image pair, further images are added using the three-point algorithm (Haralick et al., 1991). The SfM is finalized by simultaneously refining the 3D points (also called sparse point cloud) and the camera poses by a bundle adjustment step (Triggs et al., 2000).

As we aim to use the reconstruction for inspection tasks, it is important to know the reconstruction's absolute position and orientation in a specific geographic context. This can be done by georeferencing. We apply the approach of (Rumpler et al., 2014). There, the metric scale and the pose of the reconstruction are determined robustly by exploiting the GPS coordinates acquired together with the images.

According to the task of power line corridor inspection, the sparse point cloud does not provide enough information about the power line itself. Therefore, we add line features to the sparse point cloud. We follow (Hofer et al., 2016) and based on pre-aligned cameras we detect 2D line segments and match them in 3D using geometric constraints. The matching hypotheses are then verified using a multi-view approach and result in an accurate 3D reconstruction of wiry and line-based structures.

\subsection{Semantic Segmentation}

The semantic segmentation is a core element of our reconstruction approach as it influences the individual parts of the 3D reconstruction and adds the ability to analyze the final reconstruction automatically (see Figure 1). Therefore, we require pixelwise semantically labeled images. We follow the approach of (Long et al., 2014) and use a Fully Convolutional Neural Network (FCN) to get pixel-wise segmentations. The network presented in (Long et al., 2014) is adjusted to represent the number of outputs required for our task. As we are dealing with wiry objects in our input images, and thus the receptive field of the FCN of $32 \mathrm{px}$ is too big, we extend the network by adding a Conditional Random Field represented as Recurrent Neural Network (CRFasRNN) as presented in (Zheng et al., 2015). The Conditional Random Field exploits the probabilities of the FCN and refines them by taking binary constraints into account. This enhances label changes being aligned with intensity changes (edges) in the image. To further improve the results of the CRFasRNN we added an RGBtoLAB conversion to the input images of the CRFasRNN part of the network. The final network structure is depicted in Figure 2.

\subsection{Dense Image Matching}

Based on the results of the SfM we calculate stereo image pairs and perform rectification according to the epipolar geometry. Next, depth images are generated using the Discrete-Continuous Dense Image Matching approach of (Shekhovtsov et al., 2016). 


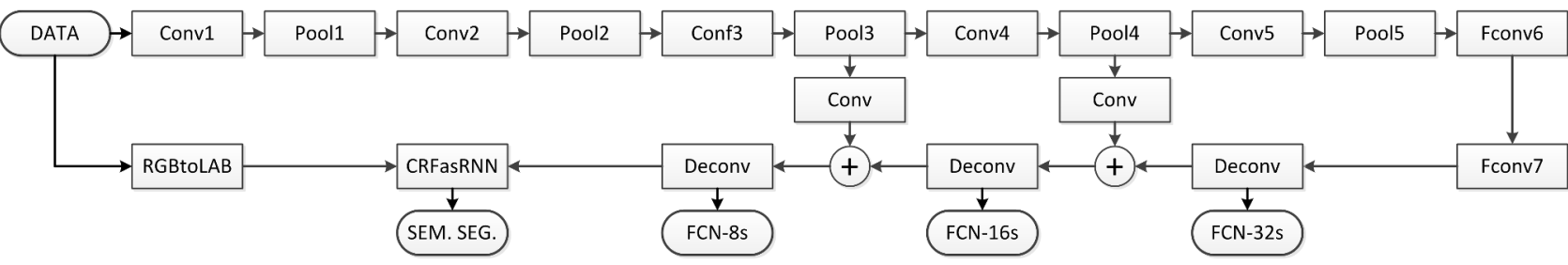

Figure 2. Schematic overview of the Neural Network: The network consists of (fully) convolutional, pooling and deconvolutional

layers. Further, the option of early exit exists, which results in coarse semantic segmentations (FCN-XXs). The pixel accurate segmentation (SEM. SEG.) is attained by adding an RGB to LAB color space conversion (RGBtoLAB) and a conditional random field represented as recurrent neural network (CRFasRNN).

First a discrete optimization problem is solved to get rough depth measurements. As this discrete optimization problem is constrained to a maximum of 128 depth steps and we are dealing with rectified stereo pairs of different baselines, we added an automatic adjustment of the step size to maximally exploit the depth range of the scene. This is done, by taking the SfM reconstruction into account. The step size is adjusted to represent the distance between the minimum and maximum depth of the sparse point cloud visible in the corresponding stereo pair within 100 depth steps. Finally, the coarse result of the discrete optimization is refined solving a continuous optimization problem exploiting the same cost function as in the discrete optimization problem.

\subsection{Depth Map Fusion with Semantic Filtering}

Based on the individual depth maps, we want to generate a dense $3 \mathrm{D}$ reconstruction. As it is known that wiry objects are not well represented in depth maps according to the regularization constraint of preserving smooth surfaces, power lines are not suited to be reconstructed using this approach. Thus, the depth maps are filtered according to the semantic segmentation. Before this filtering can be applied, the depth maps have to be transformed from the coordinate system of the rectified camera to the coordinate system of the original camera of the SfM. The required transformations have been calculated while rectification.

For the depth map fusion we use the approach presented by (Kähler et al., 2015). This approach is highly optimized for real time processing on a GPU. They model the world using a volumetrically represented signed distance function and perform raycasting for visualization. We exploit the hashing of (Nießner et al., 2013) to be unconstrained in size of the scene. To be able to use our aligned depth maps, we extended the framework of (Kähler et al., 2015) to take camera poses as input for the depth maps positioning and not to perform any tracking.

As a result we get a 3D mesh reconstruction of the surroundings. This means that the model does not contain any power lines.

\subsection{Obtaining Memory Efficiency}

Due to the high resolution of the dense mesh, the 3D reconstruction requires lots of memory and further processing would be time demanding. Thus, we rasterize the model according to a required resolution into a voxel grid and store the reconstruction in an Octomap (Hornung et al., 2013). To do so, the octree underlying the Octomap has been extended to store in addition to the occupied voxels the color, the semantic label, its color representation and the distance measurement. The usage of the extended Octomap enables to have a single model representing all visualization methods and measurements and thus save the overhead of storing the structure several times.

\subsection{D Fusion of Semantics}

The fusion of the semantics to $3 \mathrm{D}$ will enrich the $3 \mathrm{D}$ reconstructions by semantics. This is required to be able to perform automated inspection or analysis in 3D. To get robust semantics into the $3 \mathrm{D}$ reconstruction, as the $2 \mathrm{D}$ semantic segmentations may contain classification errors, we perform a histogram based voting. Based on the Octomap resulting from Section 2.5 we determine for each voxel the cameras the voxel is visible in. Then the voxel center is back-projected to these cameras and the resulting image coordinates are used to determine the label of the corresponding semantic $2 \mathrm{D}$ segmentation. Using histogram based voting the most likely label is determined and added as label to the according voxel in the Octomap.

In addition to semantically labelling the Octomap, the line reconstruction generated in Section 2.1 is filtered according to the label power line, as they have been filtered in the 3D reconstruction previously. The same histogram based voting as for the voxels is applied for each reconstructed line segment.

\subsection{Distance Evaluation}

The final step in our approach is to add the distance between power lines and trees to the $3 \mathrm{D}$ reconstruction of the surroundings represented as Octomap. As power lines are represented by line segments in our reconstruction, we evaluate the distance in $3 \mathrm{D}$ between a point and a line segment. For each voxel center $\mathbf{P}$ labeled as tree we calculate the point to line segment distance $d$ to each line segment labeled as power line and being defined by a starting point $\mathbf{V}$ and an end point $\mathbf{W}$ using:

$$
f(n)= \begin{cases}\|\mathbf{P}-\mathbf{V}\|_{2} & \text { if } t_{0} \leq 0 \\ \left\|\mathbf{P}-\left(\mathbf{V}+t_{0}(\mathbf{W}-\mathbf{V})\right)\right\|_{2} & \text { if } 0<t_{0}<1 \\ \|\mathbf{P}-\mathbf{W}\|_{2} & \text { if } t_{0} \geq 1\end{cases}
$$

where

$$
t_{0}=\frac{(\mathbf{W}-\mathbf{V}) \cdot(\mathbf{P}-\mathbf{V})}{(\mathbf{W}-\mathbf{V}) \cdot(\mathbf{W}-\mathbf{V})}
$$

The minimum distance is saved in the corresponding voxel in the Octomap.

\section{DATA ACQUISITION AND NETWORK INITIALIZATION}

In this section we will describe the hardware of the two UAVs used for image acquisition. Next, we will go into details of the acquisition itself. And finally, we will provide details on the training routine of our semantic segmentation network. 


\subsection{Hardware}

When performing power line corridor clearance distance inspection it is likely to use different acquisition platforms constrained by the surrounding area. A multi-rotor will be used in areas where power lines follow steep slopes. The use of a fixed wing UAV would be suited for power line corridors that span over large and flat areas. These constraints to the acquisition platform motivated us to use two different UAVs, a Falcon8 and an eBee to record our test sets.

The Falcon 8 has been developed by Ascending Technologies $\mathrm{GmbH}^{4}$. It is an octo-rotor equipped with a Sony NEX-5n compact camera and a $24 \mathrm{~mm}$ lens. The camera has an image resolution of $16 \mathrm{Mpx}$. The flight time of the Falcon8 is stated with 15 minutes.

The second UAV, the eBee, has been developed by SenseFly ${ }^{5}$ and is a fixed-wing UAV. It is designed to perform pre-planned regular flight patterns completely autonomous and acquires the images at fixed distance to achieve the required overlap and ground sampling distance. Its flight time is given with 40 minutes and it is able to continue the planned mission after battery swapping. This makes the eBee suitable to survey large areas. The eBee is equipped with a $12 \mathrm{Mpx}$ Canon S110.

Details on the hardware specification of the two UAVs depicted in Figure 3 are summarized in Table 1.

\begin{tabular}{|l|r|r|}
\hline Acquisition Platform & Falcon8 & eBee \\
\hline Producer & Asctec & SenseFly \\
Type & Octo-Rotor & Fixed Wing \\
Flight time [min] & 15 & 40 \\
Camera & Sony NEX-5n & Canon S110 \\
\hline
\end{tabular}

Table 1. Hardware details of the two UAVs used for data acquisition.
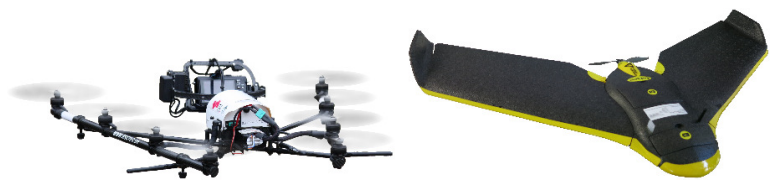

Figure 3. Image acquisition platforms used. The left UAV is the Falcon8 from Ascending Technologies $\mathrm{GmbH}$ and the right one the eBee from SenseFly.

\subsection{Dataset Acquisition}

To show the generalization of our presented pipeline we acquired two test sets using the previously described acquisition platforms. In Figure 4 the locations of the two data sets are depicted. The overview image further gives an impression of the dimensions of the areas capable to be acquired using the different UAVs.

Set1 was acquired in summer using the Falcon8. We performed a GPS assisted manual flight along the power line at a flight height of about $40 \mathrm{~m}$ above ground which results in a ground sampling distance of $12 \mathrm{~mm} / \mathrm{px}$. We recorded 387 images whereas automatic triggering of the camera was set to two seconds. A subset of the acquired images is depicted in Figure 5 left.

\footnotetext{
${ }^{4}$ www.asctec.de

${ }^{5}$ www.sensefly.com
}

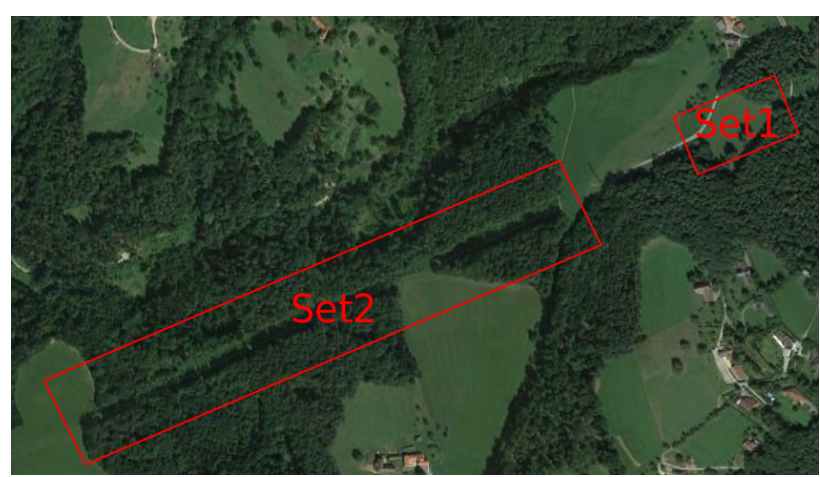

Figure 4. Overview of the test sets area: Image has been taken from Google Earth.

Set 2 was acquired in autumn using the eBee. The eBee was programmed to fly two regular grids above the ground. The first one was set to a distance to ground of $85 \mathrm{~m}$ and the second to $100 \mathrm{~m}$. The ground sampling distances are $30 \mathrm{~mm} / \mathrm{px}$ and $35 \mathrm{~mm} / \mathrm{px}$ respectively. In total 505 images were acquired and a subset is depicted in Figure 5 right.

Details of the acquired test sets are summarized in Table 2.
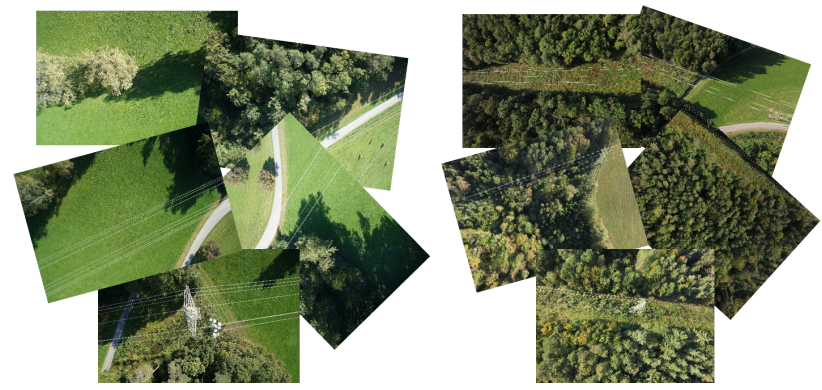

Figure 5. Comparison of acquired image sets. The differences in resolution (see power pylon) and the changes in season are visible.

\begin{tabular}{|l|r|r|}
\hline Test Set Name & Set1 & Set2 \\
\hline Acquisition Platform & Falcon8 & eBee \\
Flight Height $[\mathrm{m}]$ & 40 & $85-100$ \\
Flight Area $\left[\mathrm{m}^{2}\right]$ & 9,000 & 44,500 \\
Flight Mode & Manual Flight & Autonomous Raster \\
Season & Summer & Autumn \\
Number of Images & 387 & 505 \\
Resolution & $4912 \times 3264$ & $4000 \times 3000$ \\
\hline
\end{tabular}

Table 2. Acquisition details of the test sets

\subsection{Training the Semantic Segmentation Network}

Before any image can be segmented automatically the fully convolutional network used has to be trained. We manually annotated 25 images of Set 1 according to the classes summarized in Table 3 and further augmented them by rotation and mirroring. A final cropping to patches of $256 \times 256$ px with an overlap of $32 \mathrm{px}$ generated the training database. The cropping was required to facilitate training on the GPU. The network described in Section 2.2 has been trained in stages as described in (Shelhamer et al., 2017). First, the network was initialized using the 
weights of the fcn-32s-pascalcontext ${ }^{6}$. Next the FCN was trained for 32 s, 16 s and 8 s for 160,000 iterations respectively and finally in the last stage the CRFasRNN was added to the network and the whole network was fine-tuned end-to-end for 160,000 iterations. The parameters used for the individual training stages are summarized in Table 4.

\begin{tabular}{|l|l|l|l|}
\hline Class Name & Color & Class Name & Color \\
\hline Pylon & dark gray & Building & red \\
Tree & dark green & Street & light gray \\
Grass & light green & Clutter & magenta \\
Field & light brown & Power Line & cyan \\
\hline
\end{tabular}

Table 3. Semantic classes and the according color coding.

\begin{tabular}{|l|r|r|r|r|}
\hline Network & FCN-32s & FCN-16s & FCN-8s & CRF \\
\hline Learning Rate & $1 \mathrm{e}-10$ & $1 \mathrm{e}-12$ & $1 \mathrm{e}-14$ & $1 \mathrm{e}-14$ \\
Policy & fixed & fixed & fixed & fixed \\
Momentum & 0.99 & 0.99 & 0.99 & 0.99 \\
Weight Decay & 0.0005 & 0.0005 & 0.0005 & 0.0005 \\
Iterations & 160,000 & 160,000 & 160,000 & 160,000 \\
\hline
\end{tabular}

Table 4. Summary of the parameters used for the individual training stages.

\section{RESULTS}

In this section we present the results of our automated power line corridor inspection task according to vegetation undercut detection. First, we give results of the semantic segmentation, followed by intermediate results of the $3 \mathrm{D}$ reconstruction pipeline and finally the semantically enriched $3 \mathrm{D}$ reconstruction including color coded distance measurements between trees and power line.

\subsection{Semantic Segmentation Results}

As presented in Section 2.2 we first generate 2D semantic segmentations of the input images as semantics is required for the $3 \mathrm{D}$ reconstruction routine. An example RGB image (Figure 6a) is visualized next to the final semantic segmented image (Figure $6 \mathrm{c}$ ). To highlight the benefit of the CRFasRNN we additionally show the intermediate result of the FCN 8s (Figure 6b). It is clearly visible that the pairwise potentials of the CRF improve the boundaries of the objects, especially power lines, and the detailed structure of the pylon is segmented accurately.

\subsection{Semantic 3D Reconstruction Results}

Before we are showing the semantically enriched 3D reconstruction we depict the sparse reconstructions (Figure 7 and 8). In addition to the point cloud the reconstructed camera poses are visualized, to give an impression of the acquisition pattern. It is clearly visible, that grass is reconstructed in detail, and power lines, pylon and trees are almost missing. Thus, just a sparse reconstruction is not suited for our distance measuring task.

To enrich the reconstruction according to power lines, we use the line-based reconstruction approach of (Hofer et al., 2016) and exploit the previously aligned cameras of the sparse reconstruction. The line-based reconstruction is depicted in top- and side-view in Figure 9. Filtering the reconstructed lines according to their semantics, we are able to reconstruct just power lines.

\footnotetext{
${ }^{6} \mathrm{http} / / / \mathrm{dl}$.caffe.berkeleyvision.org/pascalcontext-fen32sheavy.caffemodel
}

(a)

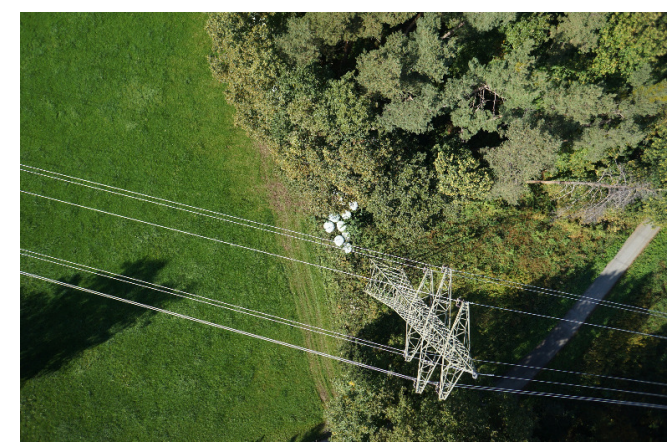

(b)

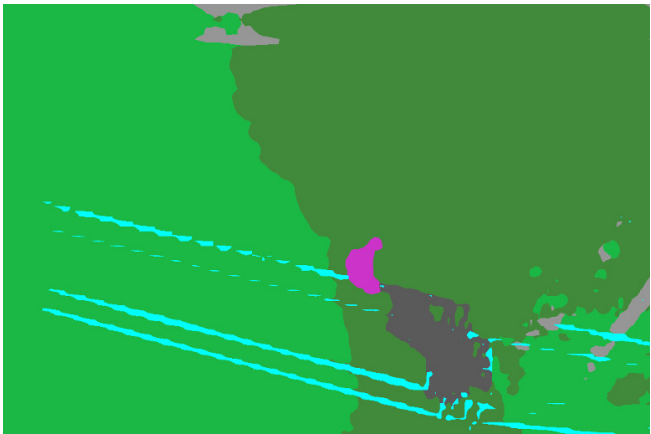

(c)

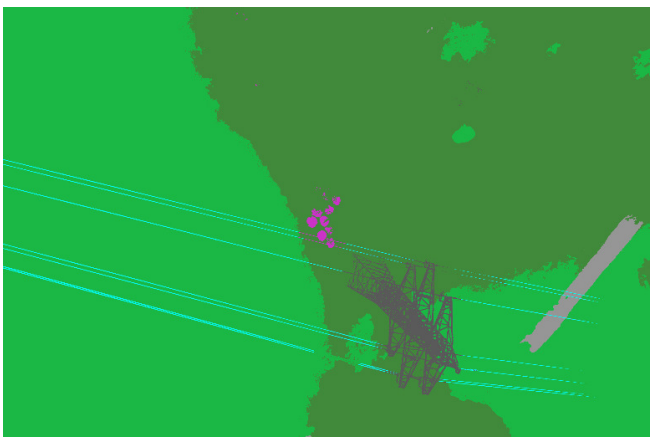

Figure 6. Example semantic segmentation result of a test image of Set1: On the top there is the reference RGB image, in the middle the intermediate result of the FCN and on the last row the final result including the CRFasRNN.

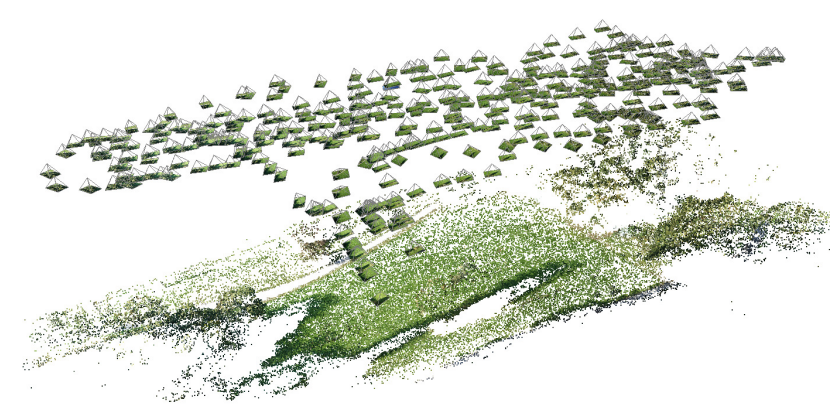

Figure 7. Sparse reconstruction result of Set1 showing the sparse point cloud and the camera poses.

An accurate reconstruction of trees is still missing. Therefore, we exploit our dense reconstruction pipeline described in Section 2.3 and 2.4. As a result we achieve a dense 3D reconstruction of the surrounding without wiry structures (power lines) as depicted in Figure 10 .

Putting everything together by simplifying the reconstruction and adding the semantics and distance measurements to the $3 \mathrm{D}$ recon- 


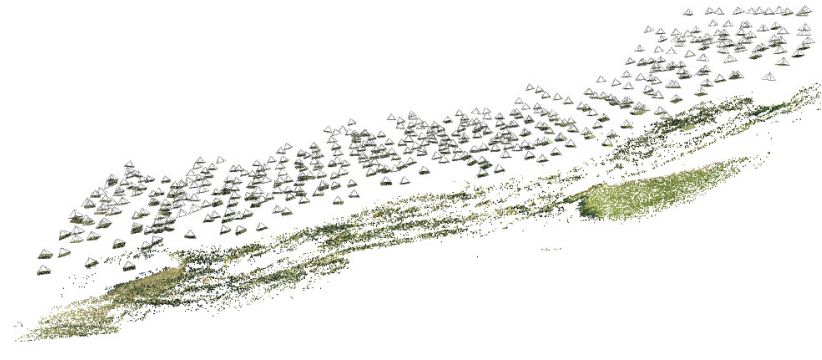

Figure 8. Sparse reconstruction result of Set2 showing the sparse point cloud and the camera poses.

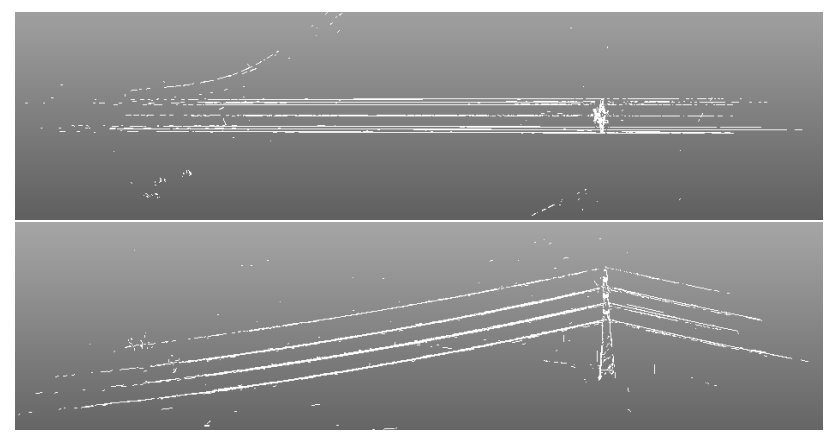

Figure 9. Reconstructed lines of Set1 depicted from top- and side-view: It can be seen, that the power lines as well as the pylon are reconstructed accurately.

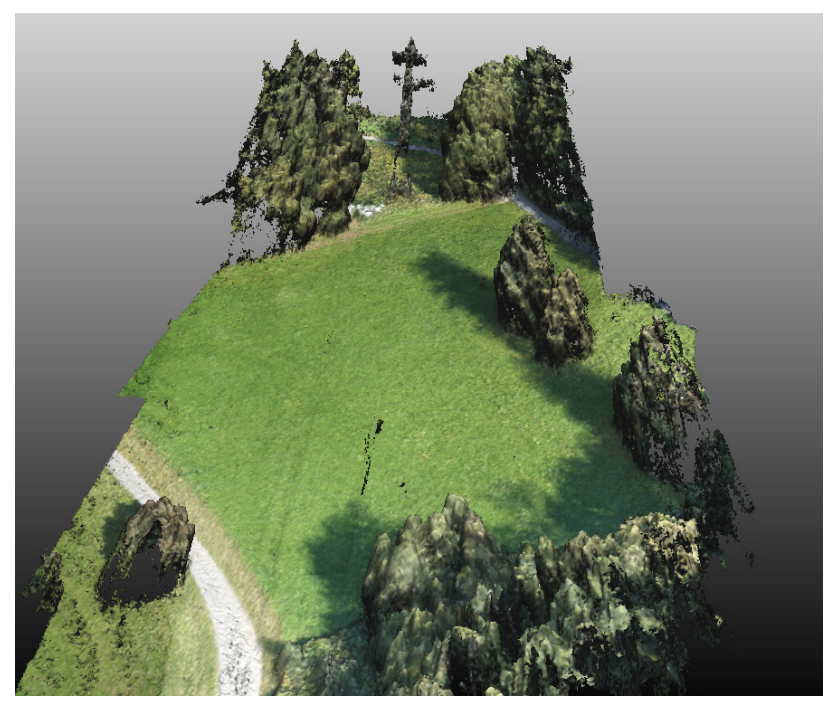

Figure 10. Dense reconstruction of Set1: It can be seen, that the vegetation is reconstructed accurately and the semantically filtered power lines are not reconstructed.

struction we get the final representation of the fully automated vegetation undercut detection as depicted in Figure 11 and 12. Due to the rasterization of the reconstruction of Set1 in voxels of $50 \mathrm{~cm}$ we gain a memory reduction by a factor of 85 .

\section{EVALUATION}

In this section we will confirm the decisions we made while setting up the automated semantic $3 \mathrm{D}$ reconstruction pipeline for vegetation undercut detection.

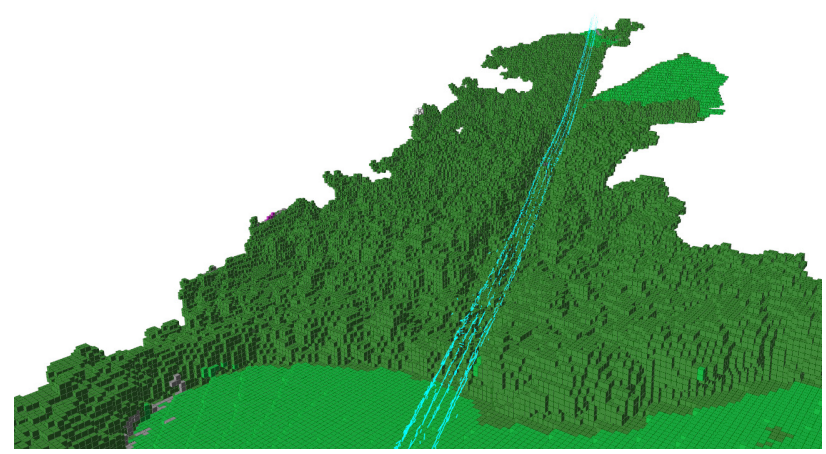

Figure 11. Color coded 3D result of Set2: The objects are color coded by their semantics according to Table 3 . The dense reconstruction has been rasterized using a voxel size of $2 \mathrm{~m}$.

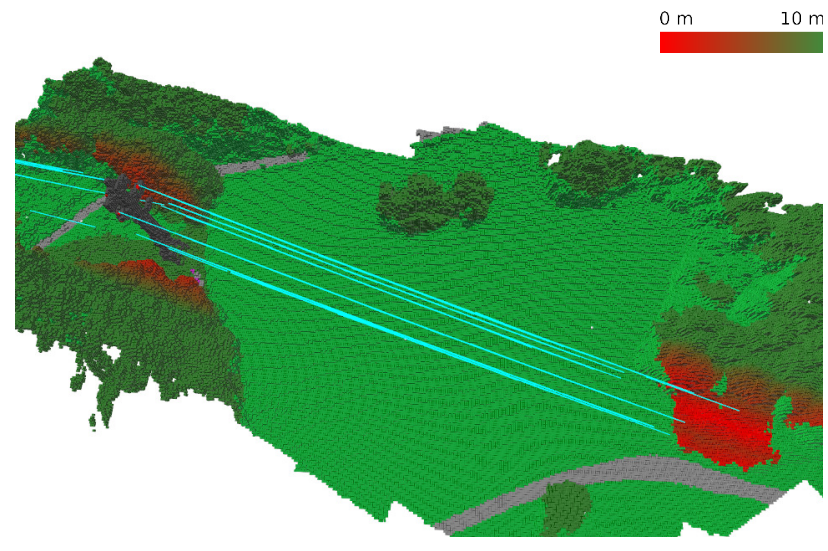

Figure 12. Color coded 3D result of Set1: The objects are color coded by their semantics according to Table 3 . In addition the distance of trees to power line is color coded from red to dark green and truncated at a distance of $10 \mathrm{~m}$.

\subsection{Reconstruct-ability of Vegetation}

To be able to perform measurements between power line and trees the vegetation has to be represented in the 3D reconstruction in detail. A typical approach to densify a sparse point cloud is to use the PMVS approach of (Furukawa and Ponce, 2010). There, a densification is achieved by local region growing based on sparse seed points. Figure 13 left shows the result of that method. Whereas this approach is well suited for man-made structures, it is clearly visible that the approach is not eligible for densification of vegetation due to the high structure and nonplanarity of vegetation. The same scene has been reconstructed using our dense reconstruction approach based on the DiscreteContinuous Dense Image Matching of (Shekhovtsov et al., 2016) and depth map fusion of (Kähler et al., 2015). We gain well reconstructed vegetation (see Figure 13 right) suitable for further inspection tasks. It is clearly visible that the trees next to the power line are completely missed by PMVS but are represented in detail using our dense reconstruction pipeline. Further, it is visible that in the dense approach the power lines are not reconstructed. The reason for that is the intentional filtering of the depth maps according to power line as they are hard to reconstruct due to the constraints of the regularization to favor smooth surfaces. This drawback is solved in our pipeline by our two lane approach of semantically separating the scene in wiry and solid objects and fusing the reconstructions in the end. 

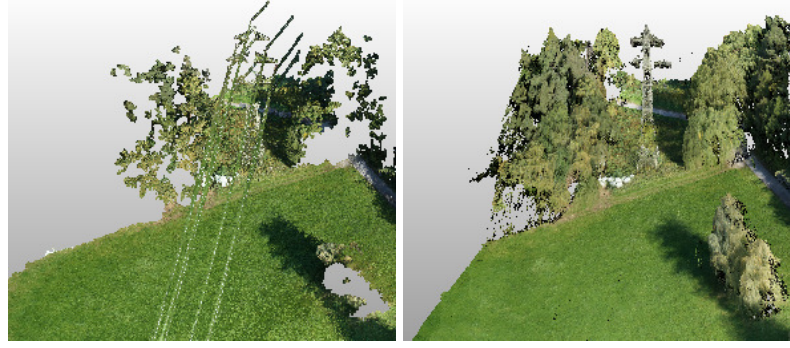

Figure 13. Comparison of 3D reconstruction methods. The left image shows a semi dense point cloud created using PMVS. It is clearly visible that the local region growing approach does not give good results for vegetation. On the right our dense 3D reconstruction pipeline provides detailed reconstruction for vegetation and thus enables further inspection tasks. Note that the power lines are not reconstructed due to the semantic filtering of the depth maps.

\subsection{Influence of Semantic on 3D Reconstruction}

In this experiment we are going to investigate the influence of the semantic filtering on depth maps. To be able to measure distances we require an accurate reconstruction. As we are dealing with dense reconstructions, we get a measurement for each pixel and due to the regularization constraint of smooth surfaces big discontinuities in depth are problematic. These discontinuities occur in the area of power lines as they are wiry objects and represented by just a few pixels (4-8 px) in the images. Figure 14 depicts such an erroneous depth map that is not able to represent the big disparity discontinuity in the vicinity of power lines. As we are dealing with a multi view reconstruction approach the area beneath the power line is depicted in different views without the disturbance and thus enables us to mask them out. This is done automatically by taking the semantic segmentation into account and masking the segmented power lines and a close area around them in the depth maps (see Figure 14 right). The fusion of the depth maps will lead to accurate $3 \mathrm{D}$ reconstructions whereas the power lines are not included any more. A comparison of a 3D reconstruction with and without filtering of the semantically segmented power lines in the depth maps is depicted in Figure 15. It is clearly visible that the filtering of power lines removes reconstruction artefacts like spikes growing toward the power line. If these artefacts are too big they would cause false alarms in the inspection task.
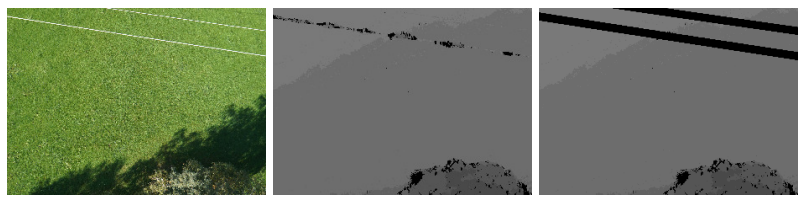

Figure 14. Generated depth maps: The left image shows the reference input image, the middle one the erroneous depth map that contains wrong depth measurements in the vicinity of power lines and the right one the filtered depth map used for fusion.

\subsection{Generalization of Semantic Segmentation}

Pixel-wise annotation of training data is a time consuming task, thus it is important that the semantic segmentation approach generalizes to different acquisition methods.
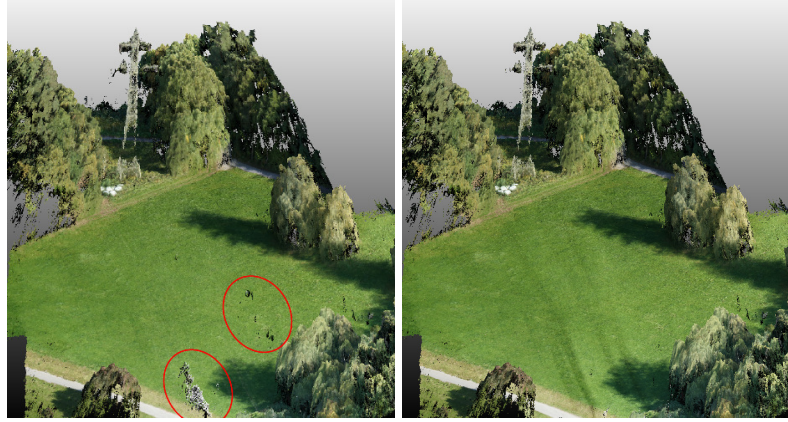

Figure 15. Comparison of 3D reconstruction: The left image shows the reconstruction without filtering the power lines and the right image the reconstruction with filtered power lines. It is

clearly visible that filtering the power lines removes

reconstruction artifacts like spikes toward the power line (marked by red ellipses).

To show the generalization of the semantic segmentation we acquired two data sets as presented in Section 3.2. Images taken from Set1 are used to train the semantic segmentation network. The trained network is then deployed on Set2. Compared to Set2, Set1 contains high resolution imagery at close distance to the power line acquired in summer. Whereas Set2 was captured in autumn using a lower resolution camera (see Figure 5 and Table 2). To demonstrate the generalization we show probability maps of the most challenging classes, power line and pylon and the reference RGB images for easy interpretation of the probability maps. Figure 16 shows in the first row the probability maps for an example taken form Set 1. The following rows give examples of Set2. As the probability maps of Set 2 result in similar probability values for the same objects it is shown that the semantic segmentation generalizes to similar scenes even acquired using different hardware and time consuming labelling, retraining or fine-tuning of the network is not required.

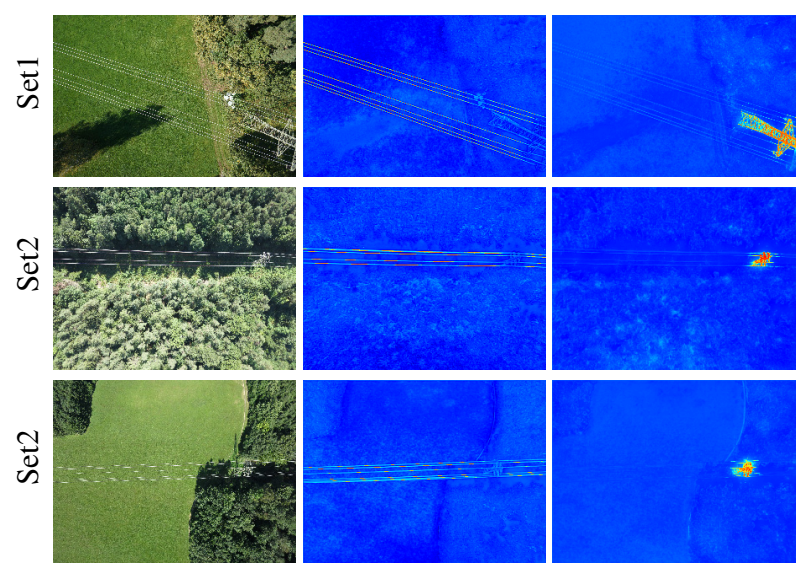

Figure 16. Generalization of semantic segmentation: The first row shows an example of a test image taken from Set1 where the network has been trained on. The further rows show test images from Set2. From left to right we start with the reference RGB image, the probability image for the class power line and the probability image for the class pylon. The warmer the color in the probability images the more likely the pixel belongs to that class. It can be seen that the probability distributions are similar and thus the semantic segmentation generalizes to Set2. 


\section{CONCLUSION}

In this paper we presented an automated pipeline to inspect vegetation undercuts of power line corridors which automatically results in a GPS-based documentation of undercut occurrences. The presented pipeline performs an automated selection of the $3 \mathrm{D}$ reconstruction method for wiry (power line) and solid objects (surrounding) using semantic segmentation based on Fully Convolutional Neural Networks. Further, an octree based data simplification routine enables to perform the inspection task and any further post-processing efficiently. Exploiting the semantically enriched 3D reconstruction an automated inspection of the vegetation undercuts of power line corridors is performed. Due to the geo-referenced semantic 3D reconstructions a documentation of areas where maintenance work has to be performed is automatically included in the measurements and can be extracted easily. We evaluated the influence of the semantic segmentation according to the $3 \mathrm{D}$ reconstruction and showed that the automated semantic separation in wiry and dense objects of the 3D reconstruction routine improved the quality of the vegetation undercut inspection. We showed that the semantic segmentation generalizes to datasets acquired using different acquisition routines and to varied seasons in time. As we described a general reconstruction and semantic segmentation approach the presented work-flow can be easily transferred to other inspection or measurement tasks.

\section{ACKNOWLEDGEMENTS}

This work has been supported by the Austrian Research Promotion Agency (FFG) project FreeLine (Bridge1/843450) and OMICRON electronics $\mathrm{GmbH}$.

\section{REFERENCES}

Daftry, S., Maurer, M., Wendel, A. and Bischof, H., 2013. Flexible and user-centric camera calibration using planar fiducial markers. In: Proceedings of the British Machine Vision Conference, BMVA Press.

Furukawa, Y. and Ponce, J., 2010. Accurate, dense, and robust multiview stereopsis. IEEE Transactions on Pattern Analysis and Machine Intelligence 32(8), pp. 1362-1376.

Haralick, R. M., Lee, D., Ottenburg, K. and Nolle, M., 1991. Analysis and solutions of the three point perspective pose estimation problem. In: Proceedings. 1991 IEEE Computer Society Conference on Computer Vision and Pattern Recognition, pp. 592-598.

Hofer, M., Maurer, M. and Bischof, H., 2016. Efficient 3d scene abstraction using line segments. Computer Vision and Image Understanding.

Hornung, A., Wurm, K. M., Bennewitz, M., Stachniss, C. and Burgard, W., 2013. Octomap: an efficient probabilistic 3d mapping framework based on octrees. Autonomous Robots 34(3), pp. 189-206.

Irschara, A., Rumpler, M., Meixner, P., Pock, T. and Bischof, H., 2012. Efficient and Globally Optimal Multi View Dense Matching for Aerial Images. ISPRS Annals of Photogrammetry, Remote Sensing and Spatial Information Sciences pp. 227-232.

Jóźków, G., Vander Jagt, B. and Toth, C., 2015. Experiments with uas for automatic modeling of power line $3 \mathrm{~d}$ geometry. ISPRS International Archives of the Photogrammetry, Remote Sensing and Spatial Information Sciences XL-1/W4, pp. 403-409.
Kähler, O., Prisacariu, V. A., Ren, C. Y., Sun, X., Torr, P. H. S. and Murray, D. W., 2015. Very High Frame Rate Volumetric Integration of Depth Images on Mobile Device. IEEE Transactions on Visualization and Computer Graphics (Proceedings International Symposium on Mixed and Augmented Reality 2015.

Long, J., Shelhamer, E. and Darrell, T., 2014. Fully Convolutional Networks for Semantic Segmentation. arXiv Prepr. arXiv1411.4038.

Lowe, D. G., 2004. Distinctive image features from scaleinvariant keypoints. Int. J. Comput. Vision 60(2), pp. 91-110.

Marcaccio, J. V., Markle, C. E. and Chow-Fraser, P., 2015. Unmanned aerial vehicles produce high-resolution, seasonallyrelevant imagery for classifying wetland vegetation. ISPRS - International Archives of the Photogrammetry, Remote Sensing and Spatial Information Sciences XL-1/W4, pp. 249-256.

Nießner, M., Zollhöfer, M., Izadi, S. and Stamminger, M., 2013. Real-time $3 \mathrm{~d}$ reconstruction at scale using voxel hashing. ACM Transactions on Graphics (TOG).

Nistér, D., 2004. An efficient solution to the five-point relative pose problem. IEEE Trans. Pattern Anal. Mach. Intell. 26(6), pp. 756-777.

Nister, D. and Stewenius, H., 2006. Scalable recognition with a vocabulary tree. In: Proceedings of the 2006 IEEE Computer Society Conference on Computer Vision and Pattern Recognition Volume 2, CVPR '06, IEEE Computer Society, Washington, DC, USA, pp. 2161-2168.

Rumpler, M., Daftry, S., Tscharf, A., Prettenthaler, R., Hoppe, C., Mayer, G. and Bischof, H., 2014. Automated end-to-end workflow for precise and geo-accurate reconstructions using fiducial markers. ISPRS Annals of the Photogrammetry, Remote Sensing and Spatial Information Sciences 2(3), pp. 135

Shekhovtsov, A., Reinbacher, C., Graber, G. and Pock, T., 2016. Solving Dense Image Matching in Real-Time using DiscreteContinuous Optimization. arXiv Prepr. arXiv1601.06274.

Shelhamer, E., Long, J. and Darrell, T., 2017. Fully convolutional networks for semantic segmentation. IEEE Transactions on Pattern Analysis and Machine Intelligence 39(4), pp. 640-651.

Triggs, B., McLauchlan, P. F., Hartley, R. I. and Fitzgibbon, A. W., 2000. Bundle adjustment - a modern synthesis. In: Proceedings of the International Workshop on Vision Algorithms: Theory and Practice, ICCV '99, Springer-Verlag, London, UK, UK, pp. 298-372.

Tscharf, A., Rumpler, M., Fraundorfer, F., Mayer, G. and Bischof, H., 2015. On the use of uavs in mining and archaeology - geo-accurate $3 \mathrm{~d}$ reconstructions using various platforms and terrestrial views. In: International Conference on Unmanned Aerial Vehicles in Geomatics, Vol. II-1/number W1, ISPRS Annals, pp. 15-22.

Zhang, J., Jung, J., Sohn, G. and Cohen, M., 2015. Thermal infrared inspection of roof insulation using unmanned aerial vehicles. ISPRS - International Archives of the Photogrammetry, Remote Sensing and Spatial Information Sciences XL-1/W4, pp. 381-386.

Zheng, S., Jayasumana, S., Romera-Paredes, B., Vineet, V., Su, Z., Du, D., Huang, C. and Torr, P., 2015. Conditional random fields as recurrent neural networks. In: International Conference on Computer Vision (ICCV). 